\title{
Predicting the risk of colonic adenomatous polyps in obese patients by TANITA: A new and practical method
}

\author{
Özlem Yönem*, Sebila Dökmetaş**, Hilmi Ataseven***, Hatice Özer****, Fatih Kılıçlı***** \\ *Professor MD, Department of Gastroenterology, Cumhuriyet University, Sivas, Turkey \\ **Professor MD, Department of Endocrinology, Medipol University, Istanbul, Turkey \\ ***Associate Professor MD, Department of Gastroenterology, State Hospital, Malatya, Turkey \\ ****Associate Professor MD, Department of Pathology, Cumhuriyet University, Sivas, Turkey \\ ***** Associate Professor MD, Department of Endocrinology, Medipol University, Istanbul, Turkey
}

Introduction

Colorectal adenoma is recognized as a precursor lesion to the majority of colorectal cancers, which allows for screening and prevention of CRCs by colonoscopic examination and polypectomy. Understanding the risk factors for colorectal neoplasm can provide guidance in developing strategies targeted toward its prevention.

The relation between body mass index and the presence of colorectal adenoma is controversial . However several studies suggest that central obesity or visceral obesity is more importantly associated with the risk of colorectal neoplasm than general obesity, which is expressed as an elevated body mass index .

The optimum technique for the accurate quantification of visceral fat is presently computed tomography (CT). However, it is not suitable for routine use in population and moreover poses the patients to ionizing radiation. On the other hand, although the waist/hip ratio and waist circumference are both cheap, safe and practical they are operator dependent and not as objective as CT.

The Tanita AB-140 (Tanita Corp, Tokyo, Japan) has the potential to provide valuable information to improve the assessment of individual health risks about obesity or to monitor the impact of intervention. It also may overcome the observer error associated with manual waist circumference.

We for the first time in the literature aimed to find out whether there is association between the obesity parameters determined by TANITA such as fat percent, body mass index and visceral fat rating and colonic polyp presence.

\section{Material and Methods}

\section{Study Participants}

We conducted a cross-sectional study using a consecutive series of subjects who underwent colonoscopy during December 2012 to September 2013 at the Cumhuriyet University Hospital in Sivas, Turkey. All of the patients had an indication for colonoscopy, the procedure was not carried out in asymptomatic patients for the purpose of screening. A consecutive series of 231 cases (123 female, 108 male) with a mean age of 50 \pm 15 (19-84 years) who underwent total colonoscopy were eligible

Colonoscopy

Colonoscopic findings were classified as normal, colorectal polyps, and non-polypoid benign lesions. During colonoscopy, the location, size and number of colorectal adenomas were recorded.

For the patients with multiple adenomas, the size and appearance of the adenoma with advanced pathology or the largest adenoma were reported.

\section{TANITA measurements}

Body mass index, fat percent and visceral fat rating of participants were determined by using the device TANITA BC 420 MA High Capacity Body Composition Analyser with integral printer (Tokyo, Japan). The manufacturers indicate that rating from1 to 12 describes healthy level of visceral fat and ratings over 13 indicate an excess level of visceral fat.

\section{Results}

Two hundred and thirty one patients underwent total colonoscopy. We detected 3 colon cancer, 63 adenomatous polyps and 169 non-polypoid benign lesions by colonoscopy. Colonoscopy was normal in 96 patients.

We divided the 231 patients into group 1 and group 2 which conferred to the patients with polyps and without polyps respectively. Number of patients in the first group was 63 while the remaining 168 cases formed the second group. Of the 63 patients with adenomatous polyps; histology revealed tubular adenoma in 43, villous adenoma in 3 and tubulovillous adenoma in 17 cases. Three patients had high-grade dysplasia pathologically. Thirty-nine (61.9\%) patients had single polyp, while 24(38.1\%) had multiple polyps. Fourteen polyps (22.6\%)were located at the proximal colon, while $36(58.1 \%)$ were located in the distal colon and $13(19.1 \%)$ of the polyps were located at both proximal and distal colon. Fifty-three $(84.1 \%)$ of the polyps had a diameter of $<1 \mathrm{~cm}$ and 10 polyps $(5.90 \%)$ had a diameter of $\geq 1 \mathrm{~cm}$.

Comparison of some parameters between group 1 (patients with polyps) and group 2 (patients without polyps) are given in Table 1. We have found that patients with colonic polyps (Group 1) were significantly older than Group 2 and also had significantly higher serum BUN, creatinine and fasting glucose levels. Visceral fat rating by TANITA was also significantly higher in patients with colonic polyps. However we found no difference between the groups by means of serum ALT, AST, triglyceride, LDL cholesterol, hemoglobin, GGT and body mass index.

Distribution of polyp presence due to gender, presence of diabetes mellitus and some TANITA parameters are given in Table 2 . The prevalance of colonic adenomatous polyps were significantly higher in male patients in diabetics and in patients having a visceral fat rating score of $>12$. According to fat percent classification by TANITA. There was a significant difference between healthy $(0)$ and overweight $(+)$ and also healthy and obese $(++)$ patients by means of polyp prevalance however there was not any significant difference between overweight and obese patients in terms of polyp prevalance. However there was no significant difference in colonic polyp prevelance of either healthy, overweight and obese patients when body mass index by TANITA.

\begin{tabular}{|c|c|c|c|}
\hline & Group 1(n=63) & Group 2(n=168) & p \\
\hline Age (Years) & $58 \pm 12$ & $47 \pm 14$ & $p<0.05$ \\
\hline ALT(U/L) & $24.19 \pm 20.38$ & $22.03 \pm 15.08$ & $p>0.05$ \\
\hline AST(U/L) & $24.98 \pm 15.12$ & $22.47 \pm 9.75$ & $p>0.05$ \\
\hline GGT & $28.50 \pm 28.41$ & $24.00 \pm 25.33$ & $p>0.05$ \\
\hline Hemoglobin & $13.95 \pm 2.20$ & $13.35 \pm 2.40$ & $p>0.05$ \\
\hline BUN & $16.71 \pm 7.01$ & $13.86 \pm 6.14$ & $p<0.05$ \\
\hline Kreatinin & $0.89 \pm 0.30$ & $0.78 \pm 0.23$ & $p<0.05$ \\
\hline Fasting glucose & $115.92 \pm 56.00$ & $101.25 \pm 28.35$ & $p<0.05$ \\
\hline Triglyceride & $141.42 \pm 89.83$ & $133.59 \pm 87.56$ & $p>0.05$ \\
\hline LDL Cholesterol & $109.77 \pm 40.07$ & $105.17 \pm 37.15$ & $p>0.05$ \\
\hline Body mass index & $28.63 \pm 5.12$ & $27.35 \pm 6.31$ & $p>0.05$ \\
\hline
\end{tabular}

Discussion

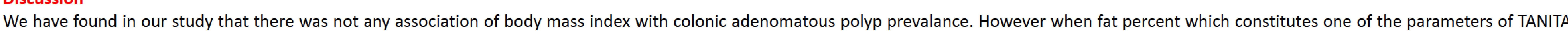

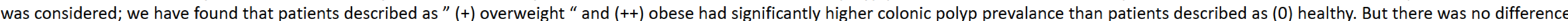

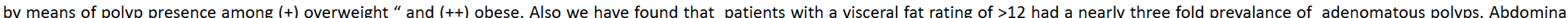

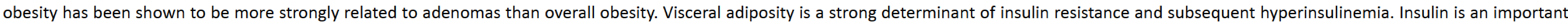
growth factor for colonic mucosal cells and colonic carcinoma cells in vitro and may have the potential to mediate the association between visceral adiposity and colorectal neoplasms

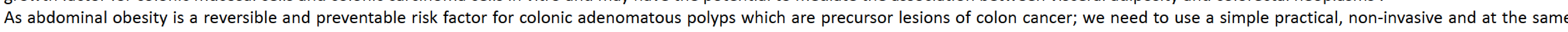

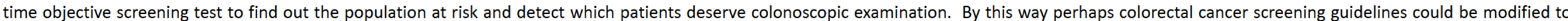

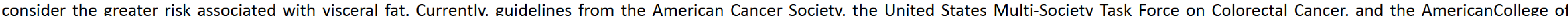

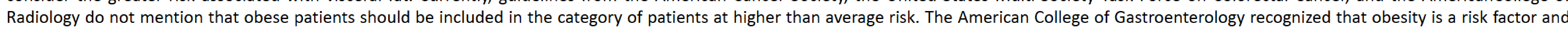
that obese patients may require earlier screening, but modification of the screening guidelines should await for the results of more studies.

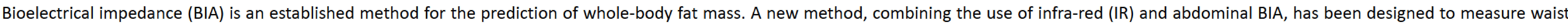

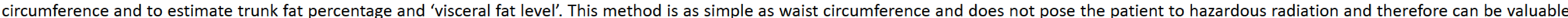

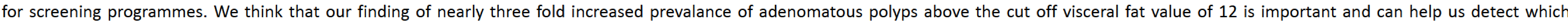
patients need screening colonoscopy.

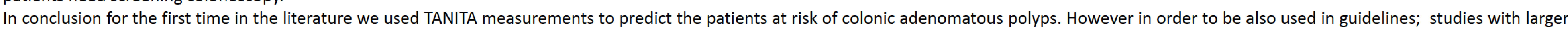
number. 